\title{
Biodiversidade de moscas-das-frutas (Diptera, Tephritoidea) em matas nativas e pomares domésticos de dois municípios do Estado do Tocantins, Brasil
}

\author{
Darcy A. do Bomfim ${ }^{1}$, Manoel A. Uchôa-Fernandes ${ }^{1}$ \& Marcos A. L. Bragança $^{2}$
}

\begin{abstract}
${ }^{1}$ Programa de Pós-Graduação em Entomologia e Conservação da Biodiversidade, Universidade Federal da Grande Dourados (UFGD), Caixa Postal 241, Rodovia Dourados-Itahum, Km 12, 79804-970 Dourados-MS, Brasil.dabomfim@yahoo.com.br; uchoa.fernandes@ufgd.edu.br ${ }^{2}$ Curso de Ciências Biológicas, Campus de Porto Nacional, Universidade Federal do Tocantins, 77500-000 Porto Nacional-TO, Brasil. marcosbr@uft.edu.br
\end{abstract}

\begin{abstract}
Biodiversity of fruit flies (Diptera, Tephritoidea) in native forests and orchards in two counties of the State of Tocantins, Brazil. This paper presents comparative and faunistic analysis of the species of fruit flies captured in McPhail traps (from June to December 2002) baited with 5\% corn protein hydrolyzed. Species richness and the patterns of population are compared between forest and orchard environments and between the counties of Palmas and Porto Nacional. A total of 1,748 individuals of Tephritidae belonging to species of three genera were collected: Tomoplagia Coquillett, 1910, Anastrepha Schiner, 1868 and Ceratitis MacLeay, 1829. Species of three genera of Lonchaeidae were also captured: Lonchaea Fallén, 1820, Neosilba McAlpine, 1962 and Dasiops Rondani, 1856. Ceratitis capitata (Wiedemann, 1824) and nineteen species of the genus Anastrepha were collected. Most of the collected individuals (69.1\%) belonged to A. obliqua (Macquart, 1935). The average numbers of tephritid individuals in Palmas and native forests were significantly lower than Porto Nacional and orchards, respectively. According to the Shannon diversity index $\left(\mathrm{H}^{\prime}\right)$ and test $\mathrm{t}$ used for comparing the fruit flies fauna among the environments, it was verified that only one comparison showed significant difference $(\mathrm{P}<0,05)$, that is, the diversity of the orchards in Palmas $(\mathrm{H}=1.96)$ was greater than in Porto Nacional $(\mathrm{H}=0.81)$. Based on the faunistic indexes A. obliqua and A. distincta Greene, 1934 were found to be constant, dominant and very abundant in almost all environments.
\end{abstract}

KEYWORDS. faunisitc analysis; faunistic index; Lonchaeidae; Tephritidae.

RESUMO. Biodiversidade de moscas-das-frutas (Diptera, Tephritoidea) em matas nativas e pomares domésticos de dois municípios do Estado do Tocantins, Brasil. Este trabalho apresenta análise faunística comparativa das espécies de moscas-das-frutas capturadas em armadilhas McPhail (junho a dezembro de 2002) com proteína hidrolisada de milho a 5\%. Foram comparadas a riqueza de espécies e a estrutura populacional entre ambientes de mata e pomar dos municípios de Palmas e Porto Nacional, TO. Foram capturados 1.748 indivíduos de espécies de três gêneros de Tephritidae: Tomoplagia Coquillett, 1910, Anastrepha Schiner, 1868 e Ceratitis MacLeay, 1829. De Lonchaeidae foram capturadas espécies de três gêneros: Lonchaea Fallén, 1820, Neosilba McAlpine, 1962 e Dasiops Rondani, 1856. Ceratitis capitata (Wiedemann, 1824). Dezenove espécies de Anastrepha foram coletadas, sendo a maioria dos indivíduos $(69,1 \%)$ de A. obliqua (Macquart, 1835). Não houve diferença significativa $(\mathrm{P}<0,05)$ do número médio de espécies coletadas entre municípios e entre ambientes, mas o número médio de indivíduos de Tephritidae do município de Palmas e do ambiente mata foram significativamente menores do que de Porto Nacional e pomar, respectivamente. Pelo índice de diversidade de Shannon (H'), utilizado para comparar a diversidade de moscas-das-frutas entre os ambientes dos dois municípios, verificou-se que apenas a diversidade do pomar de Palmas $\left(H^{\prime}=1,96\right)$ foi maior do que a do pomar de Porto Nacional $\left(H^{\prime}=0,81\right)$. Na determinação dos índices faunísticos, $A$. obliqua e A. distincta Greene, 1934 apresentaram-se geralmente como constantes, dominantes e muito abundantes.

PALAVRAS-CHAVE. Análise faunística; índice faunístico; Lonchaeidae; Tephritidae.

As moscas-das-frutas (Tephritidae e Lonchaeidae) são os insetos de maior importância econômica mundial na produção de frutas e hortaliças, uma vez que prejudicam sua qualidade e comercialização. Representam também o maior obstáculo ao livre trânsito de frutas no comércio internacional, ocupando uma posição de destaque entre as pragas da fruticultura brasileira (Duarte \& Malavasi 2000; Malavasi et al. 2000).

Inventários de espécies de moscas-das-frutas de uma determinada região são de fundamental importância para o desenvolvimento de estudos em áreas do conhecimento, como ecologia, biologia e controle (Araújo et al. 2000), além de permitir definir as espécies que representam ameaça às culturas agrícolas (Kovaleski et al. 2000). O conhecimento sobre a biodiversidade de moscas-das-frutas, em áreas nativas e cultivadas, permite também o desenvolvimento de técnicas de manejo das populações desses insetos, além de fornecer subsídios à elaboração de projetos na área de fruticultura nas diferentes regiões estudadas.

Os estudos de biodiversidade realizados com outros insetos e que comparam áreas nativas com áreas cultivadas, geralmente, mostram que a diversidade de espécies tende a ser maior em áreas nativas, e que há maior abundância de indivíduos de espécies dominantes em áreas cultivadas com monoculturas do que com policulturas (Altieri \& Letourneau 1984; Andow 1991; Bragança et al. 1998). A tendência de menor diversidade de insetos em áreas cultivadas em relação às áreas nativas, possivelmente, seja observada também no caso de comparação da diversidade de moscas-das-frutas entre pomares domésticos com áreas de matas ciliares nativas. 
Este estudo teve como objetivos conhecer e comparar a fauna de moscas-das-frutas entre pomares domésticos e matas nativas de dois municípios do Estado do Tocantins.

\section{MATERIALE MÉTODOS}

Área de estudo e coleta de dados. As coletas das moscas foram realizadas semanalmente no período de junho a dezembro de 2002, em dois municípios do Tocantins: (i) Porto Nacional ( $10^{\circ} 44^{\prime} S$ e $48^{\circ} 41^{\prime}$ W e altitude média de 422 metros), nas fazendas Capangueiro (área de mata ciliar) e Cocal (área de pomar), que se situam lado a lado, a uma distância de cerca de $50 \mathrm{~km}$ da cidade, ambas com a vegetação nativa relativamente bem conservada e; (ii) Palmas ( $10^{\circ} 21^{\prime} \mathrm{S} \mathrm{e} 48^{\circ} 7^{\prime}$ W e altitude média de 476 metros), na Chácara Arara-azul, que situa-se em uma área montanhosa dentro de um vale na região de Taquaruçú.

Em cada município, foram feitas coletas em mata ciliar e pomar doméstico. As áreas de Palmas localizam-se a aproximadamente $110 \mathrm{~km}$ das áreas de Porto Nacional. Os pomares eram bastante diversificados em ambos os municípios. O pomar de Porto Nacional apresentava 24 espécies frutíferas (manga, goiaba, jaca, laranja, mexerica, limão, abacate, café, mamão, carambola, jenipapo, cacau, coco, banana, jabuticaba, amora, ameixa, saputi, nêspera, serigüela, cajá-manga, acerola, pitanga, e sete-copas), enquanto o de Palmas com 14 espécies frutíferas comuns ao primeiro (manga, goiaba, jaca, laranja, mexerica, limão, abacate, café, mamão, carambola, jenipapo, cacau, coco, banana) mais o caju.

As coletas foram feitas com armadilhas de plástico transparente modelo McPhail, cada uma contendo como atrativo alimentar $400 \mathrm{ml}$ de solução de proteína hidrolisada de milho a 5\%, estabilizada com bórax (Bio Anastrepha ${ }^{\circledR}$ ). Foram instaladas três armadilhas para cada área de coleta, totalizando 12. Na mata de cada município, as armadilhas foram instaladas a uma distância de $150 \mathrm{~m}$ entre si, distribuídas em um transecto paralelo a um córrego. Em cada pomar, as armadilhas foram instaladas aleatoriamente com distância entre elas de $50 \mathrm{~m}$. Esta distância foi menor do que na mata porque se tratavam de pequenos pomares domésticos. As armadilhas foram presas em galhos de árvores a 1,8 m do nível do solo e vistoriadas semanalmente para a troca de atrativo e recolhimento dos insetos capturados, os quais foram armazenados em frascos com álcool $80 \%$ para transporte para o laboratório.

A triagem dos insetos foi realizada no Laboratório de Entomologia da Universidade Federal do Tocantins (UFT), Campus de Porto Nacional, TO. Após a separação, as moscas foram transportadas em frascos com álcool $70 \%$ para o Laboratório de Insetos Frugívoros da Universidade Federal da Grande Dourados (UFGD) para identificação.

O transverso analítico foi utilizado como desenho experimental, envolvendo duas variáveis de exposição (fatores): ambiente com dois níveis (pomar doméstico e mata ciliar) e município com dois níveis (Palmas e Porto Nacional), e mais duas variáveis resposta: número de indivíduos e de espécies de moscas-das-frutas. Cada parcela foi constituída pela soma do número das moscas adultas capturadas em três armadilhas por área de coleta. Para cada parcela foram realizadas 52 repetições em cada nível da variável independente, ao longo de seis meses de coletas.

Análise de dados. Foram consideradas para análise conjunta as áreas de mata ciliar e de pomar doméstico dos dois municípios, mas cada área de mata e cada área de pomar foi, também, considerada separadamente para análise. As análises estatísticas das espécies de moscas-das-frutas incluíram somente as fêmeas.

O número médio de indivíduos e de espécies obtidos entre os dois municípios e os dois ambientes foram comparados pelo test $\mathrm{t}$, visto que neste inventário, o número de repetições foi grande $(n=52)$ e igual em cada nível, o que torna esse robusto, podendo ser empregado para calcular a significância entre os resultados, independente dos dados não atenderem às condições de normalidade e de homogeneidade das variâncias (Dawson \& Trapp 2003). A análise foi realizada com nível de significância $\alpha=5 \%$.

As características de cada comunidade foram determinadas através de índices faunísticos de diversidade de Shannon (H'), eqüitabilidade (E), frequiência (absoluta e relativa), abundância, constância e dominância. A diversidade de Shannon e a eqüitabilidade foram calculados segundo as equações: $\mathrm{H}^{\prime}=$ $\sum \mathrm{p}_{\mathrm{i}} \ln \mathrm{p}_{\mathrm{i}}$ e E $=\mathrm{H}^{\prime} / \operatorname{lnS}$ (Magurram 1988). Os valores dos índices H' e E obtidos foram utilizados para se comparar a biodiversidade de moscas-das-frutas entre ambientes diferentes (mata e pomar) e iguais dos dois municípios, utilizando o teste $\mathrm{t}$.

A frequiência, porcentagem do número de indivíduos de uma espécie, em relação ao número de indivíduos total de indivíduos coletados no ambiente amostrado (Silveira Neto et al. 1976), foi calculada pela equação: $\mathrm{F}=\mathrm{n} / \mathrm{N} \times 100$, onde, $\mathrm{F}=$ frequiência $(\%), \mathrm{n}=$ número de exemplares de cada espécie coletada e $\mathrm{N}=$ número total de exemplares das espécies coletadas.

A constância, porcentagem de ocorrência das espécies em um levantamento (Silveira Neto et al. 1976), foi calculada pela equação: $\mathrm{C}=(\mathrm{P} / \mathrm{N}) x 100$, sendo: $\mathrm{C}=$ Constância, $\mathrm{P}=$ número de coletas contendo a espécie, $\mathrm{N}=$ número total de coletas realizadas. As classes foram estabelecidas através do cálculo do intervalo de confiança (IC) da média aritmética a 5\% de probabilidade. As espécies foram distribuídas nas seguintes classes: constante (w), quando a porcentagem de coletas contendo a espécie foi maior do que o limite superior do IC; acessória (y), quando a porcentagem de coletas contendo a espécie situou-se dentro do IC e acidental (z), quando a porcentagem de coletas contendo a espécie foi menor que o limite inferior do IC.

A abundância se refere ao número de indivíduos de uma determinada categoria taxonômica por unidade de área (superfície ou volume) e varia no espaço e no tempo (Southwood 1995). Foi calculada empregando-se o desvio padrão e o intervalo de confiança da média aritmética a $1 \%$ e $5 \%$ de probabilidade. Para a estimativa, foram adotadas as 
seguintes classes: raro (r), quando o número de indivíduos capturados da espécie foi menor que o limite inferior do IC a $1 \%$ de probabilidade; disperso (d), quando o número de indivíduos capturados da espécie situou-se entre os limites inferiores do IC a $5 \%$ e a $1 \%$ de probabilidade; comum (c), quando o número de indivíduos capturados da espécie situouse dentro do IC a $5 \%$ de probabilidade; abundante (a), quando o número de indivíduos capturados da espécie esteve entre os limites superiores do IC a $5 \%$ e a $1 \%$ de probabilidade; muito abundante (ma), quando o número de indivíduos capturados da espécie foi maior que o limite superior do IC a $1 \%$ de probabilidade.

O índice de dominância corresponde à proporção do total de capturas que corresponde à espécie dominante (Southwood 1995). O organismo dominante é aquele que recebe impacto do meio e adapta-se a ele (Silveira Neto et al. 1976). O limite de dominância $(\mathrm{LD})$ foi calculado pela equação: $\mathrm{LD}=(1 / \mathrm{S}) \times 100$, onde: $\mathrm{LD}=$ Limite de dominância, $\mathrm{S}=$ número total de espécies por amostra. A espécie considerada dominante foi aquela cuja frequiência absoluta era igual ou superior ao LD.

\section{RESULTADOS}

Foram coletadas 1.748 moscas-das-frutas distribuídas entre as famílias Tephritidae e Lonchaeidae (Tab. I), das quais 1.114 (63,7\%) são fêmeas, distribuidas em três gêneros de Lonchaeidae e de três gêneros Tephritidae. Anastrepha Schiner, 1868, foi o gênero com maior percentagem de adultos capturados pelas armadilhas McPhail (74,5\%), seguido por Tomoplagia Coquillett, 1910, (12,1\%). As espécies dos demais gêneros apenas representaram até $6 \%$ do total de indivíduos capturados.

As espécies de Anastrepha foram capturadas nos dois ambientes (mata ciliar e pomar doméstico) de ambos os municípios, sendo que a maioria dos indivíduos das espécies deste gênero e de Ceratitis capitata (Weidemann, 1824) foram capturados no pomar de Porto Nacional (Tab. II). No total, foram capturadas 19 espécies de Anastrepha, sendo que três destas não foram identificadas. Quase $70 \%$ dos inidivíduos capturados pertenceram à Anastrepha obliqua (Macquart,
1935), seguida por C. capitata (9,7\%) e Anastrepha distincta Greene, 1934, (9,0\%). O somatório do número de indivíduos capturados nos dois pomares (719) foi maior que o das duas matas (167).

Não houve diferença significativa entre os municípios de Palmas e de Porto Nacional no número de espécies de Anastrepha e C. capitata capturadas $(\mathrm{P}=0,218)$, nem entre matas e pomares $(P=0,220)$. Entretanto, houve diferença significativa no número de indivíduos das espécies de Anastrepha e C. capitata capturados entre os dois municípios $(\mathrm{P}=0,002)$ e entre os dois ambientes $(\mathrm{P}=0,010)$ (Tab. III).

Pelas comparações do índice de diversidade de Shannon (Tab. IV), a mata e o pomar do município de Palmas apresentaram maior diversidade do que a mata e o pomar do município de Porto Nacional, respectivamente, sendo que a diferença foi significativa apenas entre pomares. Para todas as comparações entre mata e pomar, houve tendência de maior diversidade em áreas de mata, mas sem haver diferença significativa.

Em relação aos índices faunísticos (Tab. V), A. distincta, A. obliqua e A. pickeli Lima, 1934 foram as únicas espécies caracterizadas como muito abundantes, porém as duas primeiras na mata ciliar em ambos os municípios e a segunda no pomar de Palmas. Todavia, na mata, A. distincta e A. obliqua foram constantes e dominantes, enquanto que nos pomares foram em geral comuns, com exceção de Palmas, onde foram abundantes, mas não dominantes. A maioria das espécies foi constante e algumas como A. coronilli Carrejo \& González, 1993, A. zenildae Zucchi, 1979 e C. capitata se destacaram quanto à dominância.

\section{DISCUSSÃO}

O número de gêneros de moscas-das-frutas identificados neste trabalho (6) é semelhante ao encontrado por Garcia et al. (2003) no oeste de Santa Catarina (7), mas pode ser considerado baixo quando comparado com nove gêneros registrados no Estado de Mato Grosso do Sul (UchôaFernandes et al. 2003). Os gêneros Anastrepha, Ceratitis, Neosilba McAlpine, 1962 e Dasiops Rondani, 1856, são

Tabela I. Número de indivíduos machos $\left(\sigma^{x}\right)$ e fêmeas (우) de Tephritoidea capturados em armadilhas McPhail em matas ciliares e pomares domésticos dos municípios de Palmas e Porto Nacional, Estado do Tocantins (junho a dezembro de 2002).

\begin{tabular}{|c|c|c|c|c|c|c|c|c|c|c|}
\hline \multirow{3}{*}{ Família/Gênero } & \multicolumn{4}{|c|}{ Mata ciliar } & \multicolumn{4}{|c|}{ Pomar doméstico } & \multirow{3}{*}{ Total } & \multirow{3}{*}{$\%$} \\
\hline & \multicolumn{2}{|c|}{ Palmas $^{1}$} & \multicolumn{2}{|c|}{ P. Nacional ${ }^{1}$} & \multicolumn{2}{|c|}{ Palmas $^{1}$} & \multicolumn{2}{|c|}{ P. Nacional ${ }^{1}$} & & \\
\hline & $\sigma^{x}$ & 우 & $\sigma^{x}$ & 우 & $\sigma^{x}$ & 우 & $\sigma^{x}$ & 우 & & \\
\hline \multicolumn{11}{|l|}{ Tephritidae } \\
\hline Anastrepha Schiner 1868 & 56 & 111 & 55 & 85 & 20 & 39 & 334 & 602 & 1.302 & 74,5 \\
\hline Ceratitis McLeay 1829 & 0 & 0 & 0 & 1 & 0 & 1 & 19 & 84 & 105 & 6,0 \\
\hline Tomoplagia Coquillett, 1910 & 60 & 63 & 3 & 2 & 47 & 23 & 8 & 5 & 211 & 12,1 \\
\hline \multicolumn{11}{|l|}{ Lonchaeidae } \\
\hline Lonchaea Fallén, 1820 & 9 & 42 & 0 & 3 & 7 & 17 & 7 & 24 & 109 & 6,2 \\
\hline Dasiops Rondani, 1856 & 0 & 0 & 0 & 0 & 1 & 1 & 0 & 0 & 2 & 0,1 \\
\hline Neosilba McAlpine, 1962 & 3 & 3 & 0 & 0 & 2 & 3 & 3 & 5 & 19 & 1,1 \\
\hline Total & 128 & 219 & 58 & 91 & 77 & 84 & 371 & 720 & 1.748 & 100 \\
\hline
\end{tabular}

${ }^{1}$ Dados provenientes de três armadilhas. 
Tabela II. Número de indivíduos $(\mathrm{N})$, freqüência absoluta $(\mathrm{Fe})$ e freqüência relativa (\%) de captura das espécies de Anastrepha e Ceratitis capitata, capturadas em armadilhas McPhail em matas ciliares e pomares domésticos dos municípios de Palmas e Porto Nacional, Estado do Tocantins (junho a dezembro de 2002).

\begin{tabular}{|c|c|c|c|c|c|c|c|c|c|c|}
\hline \multirow{3}{*}{ Espécie } & \multicolumn{4}{|c|}{ Mata } & \multicolumn{4}{|c|}{ Pomar } & & \\
\hline & \multicolumn{2}{|c|}{ Palmas } & \multicolumn{2}{|c|}{ P. Nacional } & \multicolumn{2}{|c|}{ Palmas } & \multicolumn{2}{|c|}{ P. Nacional } & \multicolumn{2}{|c|}{ Total } \\
\hline & $\mathrm{N}$ & $\mathrm{Fe}$ & $\mathrm{N}$ & $\mathrm{Fe}$ & $\mathrm{N}$ & $\mathrm{Fe}$ & $\mathrm{N}$ & $\mathrm{Fe}$ & $\mathrm{N}$ & $\%$ \\
\hline${ }^{1}$ Anastrepha bezzii Lima, 1934 & 1 & 1 & 3 & 2 & 0 & 0 & 3 & 2 & 7 & 0,8 \\
\hline A. fraterculus (Wiedemann, 1930) & 3 & 2 & 2 & 2 & 2 & 2 & 10 & 2 & 17 & 1,9 \\
\hline${ }^{1}$ A. montei Lima, 1934 & 1 & 1 & 1 & 1 & 2 & 1 & 0 & 0 & 4 & 0,5 \\
\hline A. coronilli Carrejo \& González, 1993 & 6 & 6 & 0 & 0 & 3 & 2 & 0 & 0 & 9 & 1,0 \\
\hline A. pickeli Lima, 1934 & 0 & 0 & 0 & 0 & 1 & 1 & 0 & 0 & 1 & 0,1 \\
\hline A. obliqua (Macquart, 1935) & 36 & 4 & 19 & 4 & 18 & 6 & 539 & 15 & 612 & 69,1 \\
\hline${ }^{1}$ A. sororcula Zucchi, 1979 & 2 & 2 & 1 & 1 & 1 & 1 & 8 & 6 & 12 & 1,4 \\
\hline A. striata Schiner, 1868 & 0 & 0 & 0 & 0 & 4 & 3 & 6 & 5 & 10 & 1,1 \\
\hline A. zenildae Zucchi, 1979 & 9 & 7 & 2 & 1 & 2 & 2 & 3 & 3 & 16 & 1,8 \\
\hline A. distincta Greene, 1934 & 14 & 7 & 49 & 8 & 6 & 4 & 11 & 5 & 80 & 9,0 \\
\hline A. turpiniae Stone, 1942 & 2 & 1 & 0 & 0 & 2 & 1 & 4 & 3 & 8 & 0,9 \\
\hline A. leptozona Hendel, 1914 & 2 & 2 & 1 & 1 & 0 & 0 & 6 & 4 & 9 & 1,0 \\
\hline${ }^{2}$ A. mucronota Stone, 1942 & 1 & 1 & 2 & 1 & 0 & 0 & 0 & 0 & 3 & 0,3 \\
\hline${ }^{1}$ A. sagittifera Zucchi, 1979 & 1 & 1 & 0 & 0 & 0 & 0 & 0 & 0 & 1 & 0,1 \\
\hline A. belenensis Zucchi, 1979 & 1 & 1 & 1 & 1 & 0 & 0 & 1 & 1 & 3 & 0,3 \\
\hline${ }^{1}$ A. amita Zucchi, 1979 & 1 & 1 & 0 & 0 & 0 & 0 & 0 & 0 & 1 & 0,1 \\
\hline Anastrepha sp.1 & 1 & 1 & 1 & 1 & 0 & 0 & 0 & 0 & 2 & 0,3 \\
\hline Anastrepha sp.2 & 1 & 1 & 1 & 1 & 0 & 0 & 2 & 2 & 4 & 0,5 \\
\hline Anastrepha sp.3 & 1 & 1 & 0 & 0 & 0 & 0 & 0 & 0 & 1 & 0,1 \\
\hline Ceratitis capitata (Wiedemann, 1824) & 0 & 0 & 1 & 0 & 1 & 1 & 84 & 13 & 86 & 9,7 \\
\hline Total & 83 & - & 84 & - & 42 & - & 677 & - & 886 & 100 \\
\hline
\end{tabular}

${ }^{1}$ Primeiro registro para a região Norte do País;

${ }^{2}$ Espécie mencionada pela primeira vez para o Brasil.

considerados os de maior importância econômica no Brasil e, provavelmente, suas espécies estão infestando hospedeiros nativos e cultivados, incluindo os presentes nos pomares domésticos e matas ciliares amostrados.

O número de espécies de Anastrepha capturadas neste estudo (19) foi semelhante ao de outros trabalhos com o mesmo tipo de armadilha, mas com variações no número de armadilhas e no período de amostragem. Canal et al. (1998) obtiveram 20 espécies em quatro municípios de Minas Gerais, sendo $A$. obliqua também a espécie mais abundante. No Estado de São Paulo, Uramoto et al. (2005) coletaram 18 espécies, sendo Anastrepha fraterculus (Wiedemann, 1930) e A. obliqua as espécies dominantes e constantes. No noroeste do Estado do Rio de Janeiro, Ferrara et al. (2005) capturarm 16 espécies de Anastrepha em quatro municípios, tendo A. obliqua como a espécie dominante em dois deles. No oeste de Santa Catarina, foram registradas 14 espécies, e $A$. fraterculus foi a dominante
(Garcia et al. 2003). Ronchi-Teles \& Silva (2005) coletaram 13 espécies de Anastrepha na região de Manaus e consideram A. obliqua predominante no Estado do Amazonas.

A porcentagem relativa dos indivíduos de A. obliqua capturados no Estado do Tocantins é superior aos resultados para a mesma espécie, nos municípios de Anastácio e Terenos, Mato Grosso do Sul (Uchôa-Fernandes et al. 2003). Naquele trabalho, em dois anos de amostragens, A. obliqua representou apenas $11 \%$ do total de indivíduos de moscas-das-frutas capturadas. Por outro lado, as espécies de Lonchaeidae apresentaram menor abundância neste trabalho do que no de Mato Grosso do Sul.

Em comparação com os dados de Thomazini et al. (2003), em dois pomares no Estado do Acre, o número de indivíduos de Anastrepha capturados no Tocantins foi baixo. Entretanto, os resultados se assemelham quanto ao fato de A. obliqua ter sido a espécie mais abundante.

Tabela III. Número (media \pm desvio padrão) de espécies de Anastrepha e Ceratitis capitata capturadas em armadilhas McPhail em matas ciliares e pomares domésticos de dois municípios do Estado do Tocantins (junho a dezembro de 2002).

\begin{tabular}{|c|c|c|c|c|c|c|c|c|}
\hline \multirow{2}{*}{ Variáveis resposta } & \multicolumn{2}{|c|}{ Município } & \multirow[b]{2}{*}{$\mathrm{t}$} & \multirow[b]{2}{*}{$P$} & \multicolumn{2}{|c|}{ Ambientes } & \multirow[b]{2}{*}{$\mathrm{t}$} & \multirow[b]{2}{*}{$P$} \\
\hline & Palmas & Porto Nacional & & & Mata & Pomar & & \\
\hline $\mathrm{N}^{\circ}$ de espécies & $1,2 \pm 1,5$ & $1,6 \pm 1,7$ & 1,24 & 0,218 & $1,2 \pm 1,5$ & $1,6 \pm 1,8$ & 1,24 & 0,220 \\
\hline $\mathrm{N}^{\circ}$ de indivíduos & $2,4 \pm 4,7$ & $14,6 \pm 26,8$ & 3,24 & 0,002 & $3,2 \pm 5,8$ & $13,8 \pm 26,9$ & 2,79 & 0,010 \\
\hline
\end{tabular}

$\mathrm{n}=52$ 
Tabela IV. Índice de diversidade de Shannon (H') para as espécies de moscas-das-frutas, capturadas em armadilhas McPhail em matas ciliares e pomares domésticos de dois municípios do Estado do Tocantins (junho a dezembro de 2002).

\begin{tabular}{|c|c|c|c|c|c|c|}
\hline Comparações & $\begin{array}{c}\mathrm{N}^{\circ} \mathrm{de} \\
\text { Espécies }\end{array}$ & $\begin{array}{c}\mathrm{N}^{\circ} \text { de } \\
\text { indivíduos }\end{array}$ & $\begin{array}{c}\text { Equitabilidade } \\
(\mathrm{E})\end{array}$ & $\begin{array}{c}\text { Índice de diversidade } \\
\text { de Shannon }\left(\mathrm{H}^{\prime}\right)\end{array}$ & $\mathrm{t}$ & $P$ \\
\hline Mata (Palmas) versus & 17 & 83 & 0,69 & 1,96 & 0,200 & 0,843 \\
\hline Mata (Porto Nacional) & 13 & 84 & 0,55 & 1,41 & & \\
\hline Pomar (Palmas) versus & 11 & 42 & 0,79 & 1,9 & 3,125 & 0,005 \\
\hline Pomar (Porto Nacional) & 12 & 677 & 0,33 & 0,81 & & \\
\hline Mata (Palmas) versus & 17 & 83 & 0,69 & 1,96 & 1,585 & 0,843 \\
\hline Pomar (Palmas) & 11 & 42 & 0,79 & 1,9 & & \\
\hline Mata (Porto Nacional) versus & 13 & 84 & 0,55 & 1,41 & 1,147 & 0,263 \\
\hline Pomar (Porto Nacional) & 12 & 677 & 0,33 & 0,81 & & \\
\hline $\begin{array}{l}\text { Mata (Palmas + Porto Nacional) } \\
\text { versus }\end{array}$ & 18 & 167 & 0,63 & 1,83 & 1,115 & 0,833 \\
\hline Pomar (Palmas + Porto Nacional) & 15 & 719 & 0,34 & 0,93 & & \\
\hline
\end{tabular}

Neste trabalho, foram encontradas cinco espécies: Anastrepha amita Zucchi, 1979, Anastrepha bezzii Lima, 1934, Anastrepha montei Lima, 1934, Anastrepha sagittifera Zucchi, 1979 e Anastrepha sororcula Zucchi, 1979, as quais não haviam ainda sido registradas na Região Norte (Silva \& Ronchi-Teles 2000). Duas das espécies encontradas: $A$. coronilli e A. belenensis Zucchi, 1979, segundo Silva \& Ronchi-Teles (2000), são exclusivas da Amazônia brasileira. O fato de estas espécies terem sido encontradas no Estado do Tocantins, amplia o conhecimento da sua área de distribuição geográfica.

A abundância de indivíduos das espécies de moscas-dasfrutas foi significativamente maior nos pomares do que nas matas, provavelmente porque os recursos (frutos) em um pomar doméstico tendem a estar disponíveis em maior quantidade do que nas matas, com isso a abundância das espécies tende a ser maior. Analisando outros trabalhos, verifica-se que em geral, as comunidades de insetos em áreas de monoculturas agrícolas e florestais apresentam baixa riqueza e grande abundância de espécies dominantes, em comparação com áreas nativas. Matas nativas apresentam maior estabilidade e heterogeneidade da vegetação, expressando elevada riqueza de espécies de insetos e maior equiitabilidade na distribuição dos indivíduos das diferentes espécies (Altieri \& Letourneau 1984; Andow 1991; Bragança et al. 1998).

O maior índice de diversidade do pomar do município de Palmas em relação ao de Porto Nacional, apesar destes ambientes possuírem riqueza de espécies semelhantes, justifica-se pelo fato de que duas espécies, A. obliqua e $C$. capitata, representaram a maioria dos indivíduos no pomar de Porto Nacional, alcançando cerca de $92 \%$ do total de indivíduos capturados. Isto, consequientemente, resultou em uma baixa equiitabilidade neste ambiente.

Na análise do índice de Shannon, observa-se que há maior diversidade nas matas em relação aos pomares. Isto pode ser explicado, em parte, pelo fato de que em ambientes naturais as comunidades, geralmente apresentam muitas espécies, representadas por relativamente poucos indivíduos, em comparação às comunidades de agroecossistemas, como os pomares (complexidade vegetal reduzida), os quais tendem a ser representadas por um grande número de indivíduos de moscas-das-frutas, distribuídos entre poucas espécies dominantes.

Apesar de em um pomar doméstico poder coexistir uma grande diversidade de espécies de plantas frutíferas, este é um ambiente que favorece o estabelecimento de espécies pragas, pois as plantas estão aglomeradas. Por outro lado, os ambientes de matas nativas oferecem diversidade de plantas de várias famílias, sendo o local com maior probabilidade de se encontrar espécies monófagas.

A maior abundância de A. obliqua no pomar de Porto Nacional indica que esta espécie é capaz de explorar grande parte dos recursos disponíveis no pomar, o qual tinha uma quantidade maior de frutos de Anacardiaceae como manga (Mangifera indica L.), serigüela (Spondias purpurea L.), cajú (Anacardium occidentale L.), além de carambola (Averrhoa carambola L.) (Oxalidaceae), que em outros Estados brasileiros são hospedeiros preferenciais dessa espécie (Alvarenga et al. 2000; Araújo et al. 2000; Uchôa-Fernandes \& Zucchi, 2000). Isso deve ter possibilitado a manutenção de uma elevada população de frugívoros no pomar de Porto Nacional. A abundância de A. obliqua (22,75 $\pm 7,20$ indivíduos) durante o período em que os frutos de espécies de Anacardiaceae estavam disponíveis nos pomares foi significativamente maior $(\mathrm{t}=2,544$ e $\mathrm{P}=0,018 ; \mathrm{n}=10)$ em relação ao período de frutificação de outros hospedeiros (4,24 $\pm 1,03$ indivíduos). A maturação dos frutos hospedeiros silvestres (Nora et al., 2000) e a sua disponibilidade (Ronchi-Teles \& Silva, 2005), são os fatores mais importantes que influenciam na ocorrência de moscas-das-frutas.

Neste estudo, as espécies que apresentaram dominância em pelo menos um ambiente foram: A. obliqua, A. distincta, $A$. zenildae e $C$. capitata, sendo que as duas últimas não foram muito abundantes. A. obliqua, espécie dominante em todos os ambientes deve ter encontrado nessa região condições de clima e hospedeiros favoráveis para o estabelecimento de altas populações. Em função de sua capacidade competitiva, pode ter deslocado as demais espécies do gênero Anastrepha. 
Tabela V. Análise faunística de espécies de moscas-das-frutas (Tephritidae) coletadas em armadilhas McPhail em matas e pomares dos municípios de Palmas e Porto Nacional, Estado do Tocantins (junho a dezembro de 2002).

\begin{tabular}{|c|c|c|c|c|c|c|c|c|c|c|c|c|}
\hline \multirow{3}{*}{ Espécies } & \multicolumn{6}{|c|}{ Matas } & \multicolumn{6}{|c|}{ Pomares } \\
\hline & \multicolumn{3}{|c|}{ Palmas } & \multicolumn{3}{|c|}{ Porto Nacional } & \multicolumn{3}{|c|}{ Palmas } & \multicolumn{3}{|c|}{ Porto Nacional } \\
\hline & $\mathrm{C}$ & $\mathrm{D}$ & A & $\mathrm{C}$ & $\mathrm{D}$ & $\mathrm{A}$ & $\mathrm{C}$ & $\mathrm{D}$ & $\mathrm{A}$ & $\mathrm{C}$ & $\mathrm{D}$ & $\mathrm{A}$ \\
\hline Anastrepha mucronota & $\mathrm{y}$ & nd & $\mathrm{c}$ & $\mathrm{y}$ & nd & $\mathrm{c}$ & 0 & 0 & $\mathrm{a}$ & 0 & 0 & 0 \\
\hline A.fraterculus & $\mathrm{y}$ & nd & $\mathrm{c}$ & $\mathrm{y}$ & nd & $\mathrm{c}$ & $\mathrm{y}$ & nd & $\mathrm{a}$ & $\mathrm{w}$ & nd & $\mathrm{c}$ \\
\hline A. montei & $\mathrm{y}$ & nd & $\mathrm{c}$ & $\mathrm{y}$ & nd & $\mathrm{c}$ & $\mathrm{y}$ & nd & $\mathrm{a}$ & 0 & 0 & 0 \\
\hline A. coronilli & $\mathrm{w}$ & $\mathrm{d}$ & $\mathrm{c}$ & 0 & 0 & 0 & $\mathrm{y}$ & nd & $\mathrm{c}$ & 0 & 0 & 0 \\
\hline A.pickeli & 0 & 0 & 0 & 0 & 0 & 0 & $\mathrm{y}$ & nd & $\mathrm{ma}$ & 0 & 0 & 0 \\
\hline A. obliqua & $\mathrm{w}$ & $\mathrm{d}$ & $\mathrm{ma}$ & w & $\mathrm{d}$ & ma & $\mathrm{w}$ & $\mathrm{d}$ & $\mathrm{c}$ & $\mathrm{w}$ & $\mathrm{d}$ & $\mathrm{c}$ \\
\hline A. sororcula & $\mathrm{y}$ & nd & $\mathrm{c}$ & $\mathrm{y}$ & nd & $\mathrm{c}$ & $\mathrm{y}$ & nd & $\mathrm{a}$ & $\mathrm{w}$ & nd & $\mathrm{c}$ \\
\hline A. striata & 0 & 0 & 0 & 0 & 0 & 0 & $\mathrm{w}$ & nd & $\mathrm{a}$ & $\mathrm{w}$ & nd & $\mathrm{c}$ \\
\hline A. zenildae & $\mathrm{w}$ & $\mathrm{d}$ & $\mathrm{a}$ & $\mathrm{y}$ & nd & $\mathrm{c}$ & $\mathrm{y}$ & nd & $\mathrm{a}$ & $\mathrm{w}$ & 0 & $\mathrm{c}$ \\
\hline A. distincta & $\mathrm{w}$ & $\mathrm{d}$ & $\mathrm{ma}$ & $\mathrm{w}$ & $\mathrm{d}$ & ma & w & $\mathrm{d}$ & $\mathrm{a}$ & $\mathrm{w}$ & nd & $\mathrm{c}$ \\
\hline A.turpinae & $\mathrm{y}$ & nd & $\mathrm{c}$ & 0 & 0 & 0 & $\mathrm{y}$ & nd & 0 & $\mathrm{w}$ & nd & $\mathrm{c}$ \\
\hline A. leptozona & $\mathrm{y}$ & nd & $\mathrm{c}$ & $\mathrm{y}$ & nd & $\mathrm{c}$ & 0 & 0 & 0 & $\mathrm{w}$ & nd & $\mathrm{c}$ \\
\hline A. bezzii & $\mathrm{y}$ & nd & $\mathrm{c}$ & $\mathrm{y}$ & nd & $\mathrm{c}$ & 0 & 0 & 0 & $\mathrm{w}$ & 0 & $\mathrm{c}$ \\
\hline A. sagittifera & $\mathrm{y}$ & nd & $\mathrm{c}$ & 0 & 0 & 0 & 0 & 0 & 0 & 0 & 0 & 0 \\
\hline A.belenensis & $\mathrm{y}$ & nd & $\mathrm{c}$ & $\mathrm{y}$ & nd & $\mathrm{c}$ & 0 & 0 & 0 & $\mathrm{z}$ & 0 & $\mathrm{c}$ \\
\hline A. amita & $\mathrm{y}$ & nd & $\mathrm{c}$ & 0 & 0 & 0 & 0 & 0 & 0 & 0 & 0 & 0 \\
\hline Anastrepha sp.1 & $\mathrm{y}$ & nd & $\mathrm{c}$ & $\mathrm{y}$ & nd & $\mathrm{c}$ & 0 & 0 & 0 & 0 & 0 & 0 \\
\hline Anastrepha sp.2 & $\mathrm{y}$ & nd & $\mathrm{c}$ & $\mathrm{y}$ & nd & $\mathrm{c}$ & 0 & 0 & 0 & $\mathrm{w}$ & 0 & 0 \\
\hline Anastrepha sp.3 & $\mathrm{y}$ & nd & $\mathrm{c}$ & 0 & 0 & 0 & 0 & 0 & $\mathrm{c}$ & 0 & 0 & 0 \\
\hline Ceratitis capitata & 0 & 0 & 0 & 0 & nd & $\mathrm{c}$ & $\mathrm{y}$ & nd & 0 & $\mathrm{w}$ & $\mathrm{d}$ & $\mathrm{c}$ \\
\hline
\end{tabular}

$\mathrm{C}=$ Constância: $\mathrm{w}=$ constante, $\mathrm{y}=$ acessória, $\mathrm{z}=$ acidental;

$\mathrm{D}=$ Dominância: $\mathrm{d}=$ dominante, $\mathrm{nd}=$ não dominante, 0 = não ocorreu;

$\mathrm{A}=$ Abundância: $\mathrm{ma}=$ muito abundante, $\mathrm{a}=$ abundante, $\mathrm{c}=$ comum, $\mathrm{d}=$ disperso, $\mathrm{r}=$ raro, $0=$ não ocorreu

Agradecimentos. Os autores agradecem à Coordenação de Aperfeiçoamento do Pessoal de Nível Superior (CAPES), pela concessão da bolsa de mestrado à primeira autora; à bióloga Rosiléia da Silva Ribeiro, pelo valioso auxílio nos trabalhos de campo; ao Prof. Dr. Odival Faccenda da Universidade Estadual de Mato Grosso do sul (UEMS), pelo auxílio nas análises estatísticas e à Fundação de Apoio ao Desenvolvimento do Ensino, Ciência e Tecnologia do Estado de Mato Grosso do Sul (FUNDECT), pelo aporte financeiro. Os autores também agradecem a dois revisores anônimos pelas sugestões pertinentes.

\section{REFERÊNCIAS}

Altieri, M. A. \& D. K. Letourneau. 1984. Vegetation diversity and insect pest outbreaks. CRC Crit. Review in Plant Sciences 2: 131-169.

Alvarenga, C. D; N. A. Canal \& R. A. Zucchi. 2000. Moscas-das-frutas nos Estados Brasileiros: Minas Gerais, p. 265-270. In: A. Malavasi \& R. A. Zucchi (ed.). Moscas-das-frutas de importância econômica no Brasil (conhecimento básico e aplicado). FAPESP-Holos, Ribeirão Preto, 327 p.

Andow, D. A. 1991.Vegetational diversity and arthropod population response. Annual Review of Entomology 36: 561-586.

Araújo, E. L; J. L. Batista \& R. A. Zucchi. 2000. Moscas-das-frutas nos Estados brasileiros: Paraíba, p. 227-228. In: Malavasi, A. \& R.A. Zucchi (ed.). Moscas-das-frutas de importância econômica no Brasil (conhecimento básico e aplicado). FAPESP-Holos, Ribeirão Preto, 327 p.

Bragança, M.; O. D. Souza \& J. C. Zanuncio. 1998. Environmental heterogeneity as a strategy for pest management in Eucalyptus plantations. Forest Ecology and Management 102: 9-12.

Canal, D. N.; C. D. Alvarenga \& R. A. Zucchi. 1998. Análise faunística de espécies de moscas-das-frutas (Dip. Tephritidae em Minas Gerais.) Scientia Agrícola 55: 15-24.
Dawson, B. \& Trapp, R. G. 2003. Bioestatística básica e clínica. $3^{a}$ edição. McGraw-Hill, New York, 348 p.

Duarte, A. L. \& A. Malavasi. 2000. Tratamentos quarentenários, p. 187192. In: A. Malavasi \& R. A. Zucchi (ed.). Moscas-das-frutas de importância econômica no Brasil (conhecimento básico e aplicado). FAPESP-Holos, Ribeirão Preto, 327 p.

Ferrara, F. A. A.; E. L. Aguiar-Menezes; K. Uramoto; P. De Marco Jr.; S. A. Souza \& P. C. R. Cassino. 2005. Análise faunística de moscasdas-frutas (Diptera: Tephritidae) da região noroeste do no estado do Rio de Janeiro. Neotropical Entomology 34: 183-190.

Garcia, F. R. M.; J. V. Campos \& E. Corseuil. 2003. Análise faunística de espécies de moscas-das-frutas (Diptera: Tephritidae) na região oeste de Santa Catarina. Neotropical Entomology 32: 421-426.

Kovaleski, A.; R. L. Sugayama; K. Uramoto \& A. Malavasi. 2000. Moscas-das-frutas nos Estados Brasileiros: Rio Grande do Sul, p. 285-290. In: A. Malavasi \& R. A. Zucchi (ed.). Moscas-das-frutas de importância econômica no Brasil (conhecimento básico e aplicado). FAPESP-Holos, Ribeirão Preto, 327 p.

Magurran, A. 1988. Ecological diversity and its measurement Croon Helm Limited Lends. Princeton, New Jersey, 178 p.

Malavasi, A.; R. A. Zucchi. \& R. L. Sugayama. 2000. Biogeografia, p. 93-98. In: A. Malavasi \& R. A. Zucchi (ed.). Moscas-das-frutas de importância econômica no Brasil (conhecimento básico e aplicado). FAPESP-Holos, Ribeirão Preto, 327 p.

Nora, I; E. R. Hichel \& H. F. Prado. 2000. Moscas-das-Frutas nos Estados brasileiros: Santa Catarina, p. 271-275. In: Malavasi, A. \& R. A. Zucchi (ed.). Moscas-das-frutas de importância econômica no Brasil (conhecimento básico e aplicado) FAPESP-Holos, Ribeirão Preto, 327 p.

Ronchi-Teles, B. \& N. M. Silva. 2005. Flutuação populacional de espécies de Anastrepha Schiner (Diptera: Tephritidae) na região de Manaus, AM. Neotropical Entomology 34: 733-741.

Silva, N. M \& B. Roncchi-Teles. 2000. Moscas-das-frutas nos Estados Brasileiros: Amapá, Amazonas, Pará, Rondônia e Roraima, p. 203 
209. In: A. Malavasi \& R. A. Zucchi (ed.). Moscas-das-frutas de importância econômica no Brasil (conhecimento básico e aplicado). FAPESP-Holos, Ribeirão Preto, 327 p.

Silveira Neto, S; O. Nakano; D. Bardim \& N. A. Villa Nova. 1976. Manual de ecologia dos insetos. Piracicaba, Editora Agronômica Ceres, $149 \mathrm{p}$

Southwood, T. R. E. 1995. Ecological mehods: with particular reference to the study of insect populations. 2 ed. London: Chapman \& Hall. 524 p.

Thomazini, J. M.; E. S. Albuquerque \& M. F. S. Filho 2003. Primeiro registro de espécies de Anastrepha (Diptera: Tephritidae) no Estado do Acre. Neotropical Entomology 32: 723-724.
Uchôa-Fernandes, M. A. \& R. A. Zucchi. 2000. Moscas-das-Frutas nos Estados brasileiros: Mato Grosso e Mato Grosso do Sul, p. 241-245 In: A. Malavasi \& R. A. Zucchi (ed.). Moscas-das-frutas de importância econômica no Brasil (conhecimento básico e aplicado). FAPESP-Holos, Ribeirão Preto, 327 p.

Uchôa-Fernandes, M. A; I. Oliveira; R. M. S. Molina \& R. A. Zucchi. 2003. Populational flutuation of frugivorous flies (Diptera: Tephritoidea) in two orange groves in the state of Mato Grosso do Sul, Brasil. Neotropical Entomology 32: 019-025.

Uramoto, K.; J. M. M. Walder \& R. A. Zucchi. 2005. Análise quantitativa e distribuição de populações de espécies de Anastrepha (Diptera: Tephritidae) no campus Luiz de Queiroz, Piracicaba, SP. Neotropical Entomology 34: 33-39. 